\title{
How accurate are forecasts of costs of energy? A methodological contribution
}

\author{
Craig Siddons*a, Grant Allan ${ }^{\dagger \mathrm{b}}$, and Stuart McIntyre ${ }^{\ddagger \mathrm{b}}$ \\ a Doctoral Training Centre in Wind Energy Systems, University of Strathclyde, \\ 99 George Street, Glasgow, G1 1RD, United Kingdom. \\ ${ }^{\mathrm{b}}$ Fraser of Allander Institute, Department of Economics, University of \\ Strathclyde, 130 Rottenrow, Glasgow, G4 0GE, United Kingdom.
}

\begin{abstract}
Forecasts of the cost of energy are typically presented as point estimates; however forecasts are seldom accurate, which makes it important to understand the uncertainty around these point estimates. The scale of the differences between forecasts and outturns (i.e. contemporary estimates) of costs may have important implications for government decisions on the appropriate form (and level) of support, modelling energy scenarios or industry investment appraisal. This paper proposes a methodology to assess the accuracy of cost forecasts. We apply this to levelised costs of energy for different generation technologies due to the availability of comparable forecasts and contemporary estimates, however the same methodology could be applied to the components of levelised costs, such as capital costs. The estimated "forecast errors" capture the accuracy of previous forecasts and can provide objective bounds to the range around current forecasts for such costs. The results from applying this method are illustrated using publicly available data for on- and offshore wind, nuclear and CCGT technologies, revealing the possible scale of "forecast errors" for these technologies.
\end{abstract}

Keywords: cost of energy; forecast accuracy; electricity, energy scenarios.

\footnotetext{
*Email: craig.siddons@strath.ac.uk

${ }^{\dagger}$ Corresponding author. Email: grant.j.allan@strath.ac.uk

${ }^{\ddagger}$ Email: s.mcintyre@strath.ac.uk
} 


\section{Highlights}

- A methodology to assess the accuracy of forecasts of costs of energy is outlined.

- Method applied to illustrative data for four electricity generation technologies.

- Results give an objective basis for sensitivity analysis around point estimates. 


\section{Introduction}

Forecasts of costs of energy technologies are often used to provide narratives for energy development, and are typically presented as a point estimate. This paper proposes a methodology to assess the accuracy of forecasts of energy costs. Our methodology considers comparable data on historic cost forecasts and matched "contemporary estimates" to generate "forecast errors" ${ }^{1}$. In addition, quantifying these errors will provide a range around point estimates of costs, which are important inputs to policymaking as well as in empirical work (e.g. using Integrated Assessment Models (IAMs)). This would provide an objective basis for understanding the accuracy of point estimates and for guiding sensitivity analysis.

IAMs have been extensively used to develop energy scenarios for countries and regions across the world (Edenhofer et al. 2013). Such models are particularly useful as (among other factors) they include economic criteria of each technology option to form energy scenarios which are endogenous to the model (Krey \& Clarke 2011). Despite this, IAMs have some limitations which have been previously noted, for instance not capturing the heterogeneous aspect of electricity from different technologies (Edenhofer et al. 2013). A number of detailed elements affecting the future supply of energy are included in these models, including (but not limited to) future values of costs for different technologies. IAMs typically focus on long term scenarios e.g. greater than 25 years, and make use of a given set of assumptions, including point estimates for costs of different technologies.

Comparisons across energy costs, such as levelised cost of energy (LCOE) ${ }^{2}$, are often used to inform a government's stance on particular technologies, such as the level of

\footnotetext{
${ }^{1}$ We use the term contemporary estimate throughout this paper to refer to the known cost for a generation technology in a specific year. Our use of this terminology is consistent with e.g. Gross et al. (2013). Where there is more than one unit of a generation technology developed in a year, publicly available data are likely to represent the average for that technology in that country, see Grubler (2010), although this would not necessarily be the case for the use of these methods with proprietary data. We thank an anonymous referee for requesting this clarification.

${ }^{2}$ This is the "the discounted life-time fixed and variable cost of a generation technology in euro/MWh" (Edenhofer et al. 2013, p. S17)
} 
financial support a technology may require to be "competitive" with other technologies (Allan et al. 2011). Some recent work has attempted to refine the costs for technologies which might be used in IAMs by, for example, estimating "system-level" costs for variable technologies (Gross et al. 2007), while others assess the marginal economic value of changes in generation technologies (Borenstein 2012). Each of these approaches however, produce "point estimates" for the costs of each technology, and do not capture the possible range within which the future costs will lie, i.e. they ignore the issue of forecast errors.

Section 2 introduces the method, which has its origins in the assessment of economic forecasts (Granger 1996), discusses the alternative measures of costs to which the methodology could be applied and the data used for the empirical application in this paper. Our methodology could be applied to any energy cost, including capital or fuel costs, however due to data availability the empirical example we use in this paper uses the LCOE for four technologies. The empirical results from the illustrative example give an indication of the accuracy of previous forecasts and objective bounds to the range of uncertainty around current forecasts of LCOE. The results from this illustrative application using LCOEs are set out briefly in Section 3, before a discussion of the results and method in Section 4. Section 5 sets out conclusions and directions for future research, including applying the proposed methodology against other measures of costs.

\section{Methods}

\subsection{Measures of forecast accuracy}

There is a substantial literature on techniques for assessing the accuracy of economic forecasts. This literature, for example, has shown the accuracy of forecasts made by private organisations compared to public organisations, the accuracy of forecasts produced by different multinational organisations (e.g. Granger (1996), Pons (2000), Loungani 
(2001)) and the accuracy of forecasts over different horizons, i.e. the duration between when the forecast is made and the point of time to which the forecast relates (e.g. Ashiya (2006), Allan (2011)). This literature has developed a number of empirical measures that can be used to gauge the accuracy of a forecast, including the mean absolute error (MAE), root mean square error (RMSE), root mean square percentage error (RMSPE) and mean absolute percentage error (MAPE).

In our application, the MAE for each generation type $g$ can be calculated as the absolute mean of all "forecast errors", i.e. the distance between the forecast and the "contemporary estimate" of the cost. In Equation 1, $y_{p-h}^{f}$ is the forecasted cost relating to year $p$ made at a forecast horizon of $h$ years, e.g. the cost for onshore wind in 2010 made in 2005, where $h=5 . y_{g p}^{a}$ is the contemporary estimate of the cost for technology $g$ in period $p$, and $N$ the total number of paired cost forecasts and contemporary estimates for technology $g$. The further from the date of the forecast that the forecast is evaluated, one might expect errors in the forecast to be larger. Therefore we might want to produce evaluations for each forecast horizon, $h$, e.g. $5,6,7, \ldots, \mathrm{k}$ years. This being the case, for each $h$, we would calculate ${ }^{3}$ :

$$
M A E_{g}=\frac{1}{N} \sum_{n=1}^{N}\left|y_{p-h}^{f}-y_{p}^{a}\right|
$$

The root mean square error (RMSE), when compared to the MAE, places slightly more emphasis on larger errors due to the squaring process, as seen in Equation 2. This can be useful for forecasters or analysts who are concerned about larger errors.

$$
R M S E_{g}=\sqrt{\frac{1}{N} \sum_{n=1}^{N}\left(y_{p-h}^{f}-y_{p}^{a}\right)^{2}}
$$

Percentage (or proportional) errors are an alternative measure of the accuracy of

\footnotetext{
${ }^{3}$ An alternative approach, although notationally messier, would be to introduce a new subscript in Equation 1 denoting the forecast horizon being evaluated.
} 
forecasts. These take into account the scale of errors relative to the contemporary estimates and the Root Mean Squared Proportional Error (RMSPE) and Mean Absolute Proportional Error (MAPE) are shown in Equations 3 and 4.

$$
\begin{gathered}
R M S P E_{g}=\sqrt{\frac{1}{N} \sum_{n=1}^{T} \frac{\left(y_{p-h}^{f}-y_{p}^{a}\right)^{2}}{y_{p}^{a}} \times 100 \%} \\
M A P E_{g}=\frac{1}{N} \sum_{n=1}^{N} \frac{\left|y_{p-h}^{f}-y_{p}^{a}\right|}{y_{p}^{a}} \times 100 \%
\end{gathered}
$$

\subsection{What cost measure is appropriate?}

In principle, the methods outlined above can be used to calculate and analyse forecast errors for any energy costs for which there are forecasts and contemporary estimates, i.e. a contemporary estimate for a given year of a forecasted cost for a specific technology, made in a year prior to which the forecast relates). The precise cost figure to which the method can be applied is likely to depend upon the availability of comparable data. For example, capital costs (i.e. including development, construction and installation) would appear to be a useful measure. For many technologies, these will be a significant portion of total project costs. However, forecast and contemporary estimates of capital costs can be difficult to find in the public domain, with both typically known only to the developers of that technology. We return to this point in Section 4.

In the absence of such proprietary data, an alternative cost figure - and what we use in the empirical example which follows - is the LCOE. As described above, these are widely reported, and so easier to access the matched forecast and contemporary estimates that our methodology requires. There are some issues however in using LCOEs; 
specifically they are based on an aggregation of individual cost elements, such as capital and fuel costs, as well as production data. If there were forecast and outturn values for each element, the analyst could decompose the LCOE "forecast errors" in terms of the contribution of its individual components. This may be insightful, as elements within the LCOE could differ between their forecast and outturn values in ways which offset, or exaggerate their individual errors. For example, (Harris et al. 2013, p. 440) noted that observed increases in construction cost estimates can have a "dramatic" effect on the estimated future LCOE. However, in the absence of comparable forecast and outturn data either for all components of the LCOE or just capital costs, in the empirical illustration of the methodologies which follows, we use LCOE as our measure of costs $^{4}$.

\subsection{Data}

In order to implement this approach we require two types of cost data; forecasts of the costs of energy and out turn or realised costs of energy (which we refer to as "contemporary estimates" in this paper). In the illustrative empirical exercise which follows we use forecast data on the levelised costs of energy from International Energy Agency (1989, 1992, 1998, 2005, 2010). While these IEA reports provide forecasts of the levelised cost of energy, and its components (e.g. capital cost, fuel cost etc), they provide no information on the "contemporary estimates" for any of these costs.

The IEA presents its LCOE forecasts based on the forecasts it receives from participating countries, thus producing a range of estimates for multiple technologies for a single year and at a given forecast horizon. Table 1 shows the year of publication of each report matched with the forecast year for that report; it can be seen that the forecast horizon varies from five to a maximum of eight years from the publication of each report. The forecasts for levelised costs used in this paper for a given year were

\footnotetext{
${ }^{4}$ We thank a referee for drawing our attention to this empirical challenge with using the LCOE measure.
} 
obtained by averaging the costs across EU countries for each technology for the year to which the forecasts relate. This gives us a single forecast estimate for generation type $g$ in year $p$, which was produced in year $p-h$. Thus while country specific evaluations are desirable when implementing this approach in practice, for this illustrative application we collapse cross country variation into an EU average for each technology.

\section{[Table 1 here]}

We obtained our "contemporary estimates" of levelised costs of energy from Gross et al. $(2013)^{5}$. This report provides "contemporary estimates" of the total levelised costs of energy for the technologies identified earlier, but does not provide estimates for individual cost components such as capital costs, fuel costs, etc. It is for this reason the analysis which follows is undertaken using levelised costs of energy, rather than individual cost components. It is worth noting that while Gross et al. (2013) does contain forecast estimates for the levelised costs of energy, these relate to the post 2010 period for which we have no "contemporary estimates", as yet. For some technologies, contemporary estimates of the LCOE were not available for the forecast year, and so were linearly interpolated from data for the preceding and following year; again given the illustrative nature of this empirical exercise this seems a reasonable approach.

The levelised costs for both forecast and contemporary estimates utilised a $10 \%$ discount rate and all currencies were rebased to 2011 GBP using the UK governments GDP deflators (HM Treasury 2013).

[Figure 1 here]

Figure 1 shows the quantity of data used for the analysis. In all cases, the total number of paired forecast and actual values for each generation type does not exceed

\footnotetext{
${ }^{5}$ These data, in turn, come from a combination of sources, specifically Mott Macdonald $(2010,2011)$ and ARUP (2011). The authors of the paper are very grateful to the authors of this report for sharing the data underpinning their report. Errors and omissions in the use of the data however remain our responsibility.
} 
three. Due to this small sample size, the results can only be considered illustrative of the value of the methodology for assessing the accuracy of forecasts of levelised costs of energy.

\section{Results}

\subsection{Absolute errors}

Figure 2 shows the results of the MAE for each generation type across all matched forecasts and actual values of the levelised cost of energy. As expected, the largest MAE is for offshore wind technology at greater than $£ 50 / \mathrm{MWh}^{6}$. CCGT and Nuclear have fairly similar errors of around $£ 20 / \mathrm{MWh}$, while onshore wind has the smallest MAE. Figure 3 shows the RMSE. Only subtle differences can be seen compared to Figure 2.

[Figures 2 and 3 here]

\subsection{Percentage errors}

Figure 4 shows the results of the MAPE calculations. The larger observed "errors" are present for Nuclear and CCGT technologies, while - due to the errors being rescaled by the absolute value of the LCOE forecast - offshore wind returns a smaller proportionate error.

[Figure 4 here]

Figure 5 shows the RMSPE for each technology. These results are similar to the MAPE, however the offshore wind error is larger due to the presence of one larger error. As already mentioned in Section 2 the RMSPE places greater emphasis on larger, single errors.

[Figure 5 here]

\footnotetext{
${ }^{6}$ Recall, all values are reported in 2011 prices.
} 


\section{Discussion}

\subsection{Implications of results}

The illustrative results presented in Section 3 reveal that Offshore wind showed the greatest MAE, RMSE and RMSPE while the MAPE was greater for Nuclear and CCGT. These results suggest that technologies considered "mature", such as nuclear and CCGT, display forecast errors which are higher than both on- and offshore wind in proportionate terms. The finding that there are also quantifiable MAEs in forecasts of levelised costs of between $£ 15 / \mathrm{MWh}$ and $£ 25 / \mathrm{MWh}$ for "mature" technologies like onshore wind, CCGT and nuclear for a relatively short forecast horizon - one that is far shorter than the long term typically considered in IAM scenarios - may be important for the range of sensitivity analysis that can be carried out using IAMs.

Technologies in their infancy typically suffer from an apparent paradox: at this early stage in the development of the technology, policy makers will place great emphasis on having cost forecast. Unfortunately, this is when the predictions are often at their weakest due to a lack of information on the learning rates in the development of these technologies. It is also possible that past forecast errors will not necessarily be a good proxy for future errors. One obvious example of this is where there are structural breaks in key markets, for example with the oil shocks of the 1970s. Such events may lead to errors estimated using current data to underestimate future forecast errors ${ }^{7}$.

\subsection{Empirical limitations}

All of the data used in the illustrative application utilises secondary sources, and was not collected for the sole purpose of this study. In some years the data suffers from a lack of data points, and in a few cases relevant contemporary estimates were linearly

\footnotetext{
${ }^{7}$ Nevertheless, we argue that past forecast errors do provide an objective guide for sensitivity analysis around point estimates, and that this information can be useful in evaluations (e.g. using IAMs). We thank a referee for alerting us to this issue.
} 
interpolated from the surrounding years. Overall the total volume of data is not large; ideally there would be a considerable number of contemporary estimates to match to more detailed forecasts, with numerous different (and conceivably longer) forecast horizons over which to assess the accuracy of these forecasts of future costs (as presented in Table 1).

Future empirical applications of these methods are likely to be able to utilise a more extensive database than we have access to at the moment, in particular access to proprietary "contemporary estimates" would allow a more detailed comparison of cost forecasts with realised costs. In particular, analysis of the forecast errors for each of the components of levelised costs would be an interesting area of future work. However, and largely for reasons of commercial confidentiality, such data are very difficult to access. Nevertheless, when using non-proprietary data, it would be desirable to have actual outturn data.

The only study we are aware of that provides data on the components of the outturn costs is Koomey \& Hultman (2007), in addition to focussing on only one technology and one country, the data released related to construction projects that took place, in some cases, up to 35 years before. These data relate only to Nuclear power and only to the US and as such are not compatible with any of the forecast data that we use here. The paucity of data on the outturn values of these costs suggests that this is an important area of future research, particularly given that the IEA produce forecasts of these cost components.

\section{Conclusion and policy implications}

This paper has presented a methodology to assess the accuracy of forecasts of costs of energy. Our methodology requires historical data on forecast and "outturn" costs of energy, which would ideally be available for different technologies, and covering a 
range of (short and long-term) forecast horizons. The methodology outlined here was demonstrated using data on the levelised cost of energy for Nuclear, CCGT and Onand Offshore Wind, while the methodology could in principle be applied to any forecast cost which has comparable outturn data.

We find that, in line with expectations, offshore wind forecasts have larger mean absolute errors. Percentage errors proved to be different however; "mature" technologies such as CCGT and nuclear had the greater errors when compared to wind technologies. Concerns about the data used have been thoroughly addressed, and additional applications and testing of the methodology with a more comprehensive dataset is recommended. The data would preferably be primary data, cover a range of energy technologies and be consistent with forecasts of LCOEs for a range of forecast horizons.

The output of this methodology, in addition to its usefulness in giving some context to point estimates used by policy-makers, has the potential to improve the output of analytical work in this area (e.g. using IAMs) by enabling an objective understanding of the likely range of LCOE forecast errors (or as discussed earlier, for other measures of costs of energy). More fundamentally, it would be interesting to use the scale of "errors" from this analysis in applied models like IAMs to understand the importance of uncertainty surrounding forecasts of technology costs in energy scenarios.

\section{Acknowledgements}

Craig Siddons acknowledges funding from the Engineering and Physical Sciences Research Council to the Doctoral Training Centre in Wind Energy Systems, and Grant Allan acknowledges funding from the Scottish Government through the ClimateXChange programme. The opinions in the paper however are the sole responsibility of the authors and not necessarily those of the Scottish Government. 


\section{Bibliography}

Allan, G. (2011), 'How wrong were we? The accuracy of the Fraser of Allander Institutes forecasts of the Scottish economy since 2000', Fraser Economic Commentary 35(2), 45-53.

Allan, G., Gilmartin, M., McGregor, P. \& Swales, K. (2011), 'Levelised costs of wave and tidal energy in the UK: Cost competitiveness and the importance of "banded" Renewables Obligation Certificates', Energy Policy 39(1), 23-39.

ARUP (2011), 'Department of Energy and Climate Change Review of the generation costs and deployment potential of renewable electricity technologies in the UK Study Report'.

Ashiya, M. (2006), 'Are 16-month-ahead forecasts useful? a directional analysis of japanese GDP forecasts', Journal of Forecasting 25(3), 201-207.

Borenstein, S. (2012), 'The private and public economies of renewable electricity generation', The Journal of Economic Perspectives pp. 67-92.

Edenhofer, O., Hirth, L., Knopf, B., Pahle, M., Schlömer, S., Schmid, E. \& Ueckerdt, F. (2013), 'On the economics of renewable energy sources', Energy Economics 40, S12S23.

Granger, C. W. (1996), 'Can we improve the perceived quality of economic forecasts?', Journal of Applied Econometrics 11(5), 455-473.

Gross, R., Candelise, C., Heptonstall, P., Greenacre, P., Jones, F. \& Castillo, A. C. (2013), Presenting the Future: An assessment of future costs estimation methodologies in the electricity generation sector, Technical report, UKERC.

Gross, R., Heptonstall, P., Leach, M., Anderson, D., Green, T. \& Skea, J. (2007), 'Re- 
newables and the grid: understanding intermittency', Proceedings of the ICE-Energy 160(1), 31-41.

Grubler, A. (2010), 'The costs of the french nuclear scale-up: A case of negative learning by doing', Energy Policy 38(9), 5174-5188.

Harris, G., Heptonstall, P., Gross, R. \& Handley, D. (2013), 'Cost estimates for nuclear power in the UK', Energy Policy 62, 431-442.

HM Treasury (2013), 'GDP deflators at market prices, and money GDP'.

URL: https://www.gov.uk/government/statistics/gdp-deflators-at-market-prices-andmoney-gdp-march-2013

International Energy Agency (1989), Projected costs of generating electricity from power stations for commissioning in the period 1995-2000, Technical report.

International Energy Agency (1992), Projected Costs of Generating Electricity, Technical report.

International Energy Agency (1998), Projected Costs of Generating Electricity, Technical report.

International Energy Agency (2005), Projected Costs of Generating Electricity, Technical report.

International Energy Agency (2010), Projected Costs of Generating Electricity, Technical report.

URL: http://www.oecd-ilibrary.org/energy/projected-costs-of-generating-electricity2010_9789264084315-en

Koomey, J. \& Hultman, N. E. (2007), 'A reactor-level analysis of busbar costs for US nuclear plants, 1970-2005', Energy Policy 35(11), 5630-5642. 
Krey, V. \& Clarke, L. (2011), 'Role of renewable energy in climate mitigation: a synthesis of recent scenarios', Climate Policy 11(4), 1131-1158.

Loungani, P. (2001), 'How accurate are private sector forecasts? cross-country evidence from consensus forecasts of output growth', International Journal of Forecasting $\mathbf{1 7}(3), 419-432$.

Mott Macdonald (2010), 'UK Electricity Generation Costs Update', (June).

Mott Macdonald (2011), Costs of low-carbon generation technologies Committee on Climate Change Costs of low-carbon generation technologies, Technical Report May.

Pons, J. (2000), 'The accuracy of IMF and OECD forecasts for G7 countries', Journal of Forecasting 19(1), 53-63. 


\section{Tables}

Table 1: Forecast horizons for all data contained within the IEA reports International Energy Agency (1989, 1992, 1998, 2005, 2010).

\begin{tabular}{ccc} 
Published Year & Forecast year & Forecast Horizon (years) \\
\hline \hline & & \\
1989 & 1995 & 6 \\
1992 & 2000 & 8 \\
1998 & 2005 & 7 \\
2005 & 2010 & 5 \\
2010 & 2015 & 5 \\
& & \\
\hline
\end{tabular}




\section{$7 \quad$ Figures}
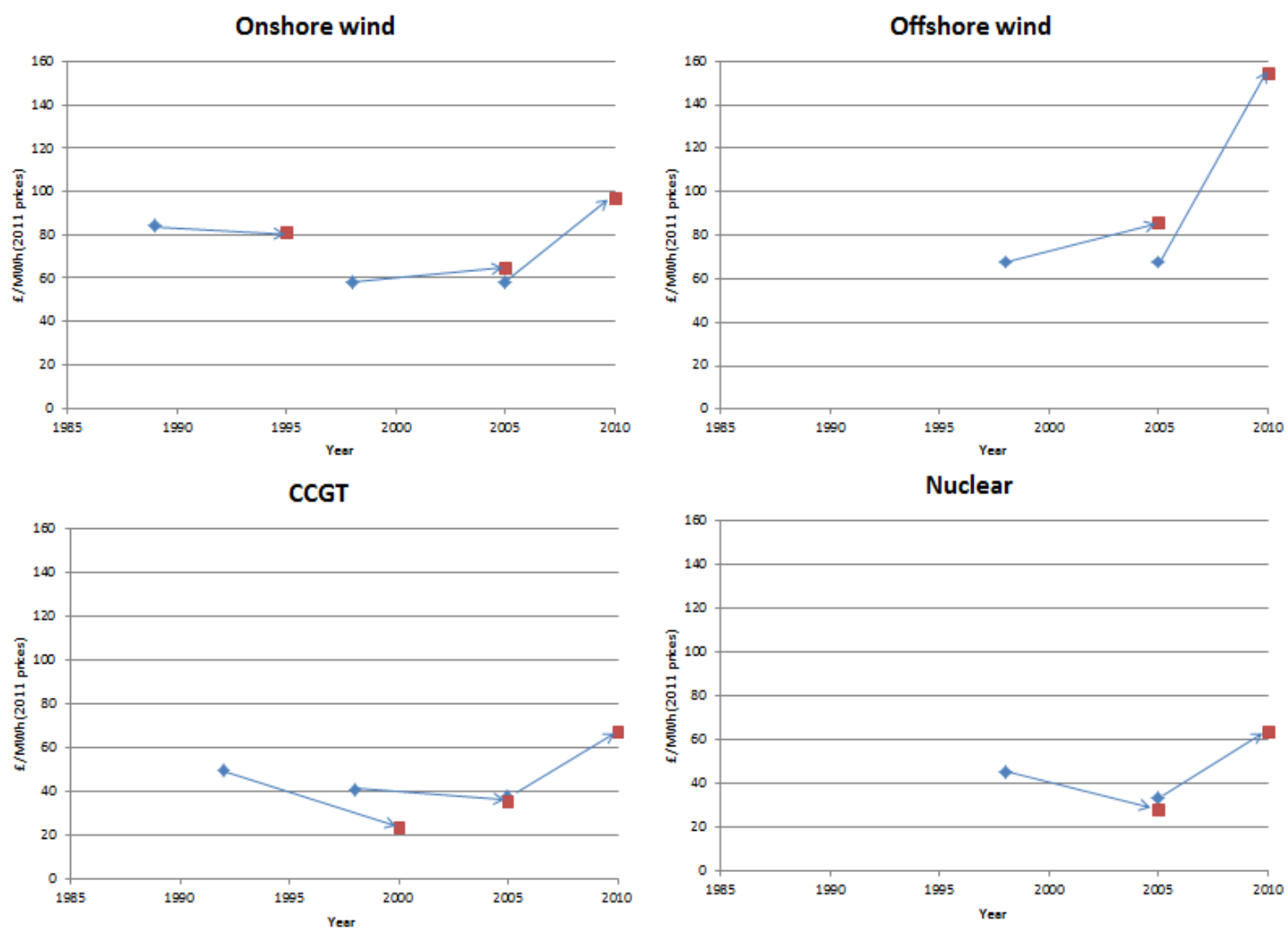

Figure 1: Summary of the usable forecast and contemporary estimate data 


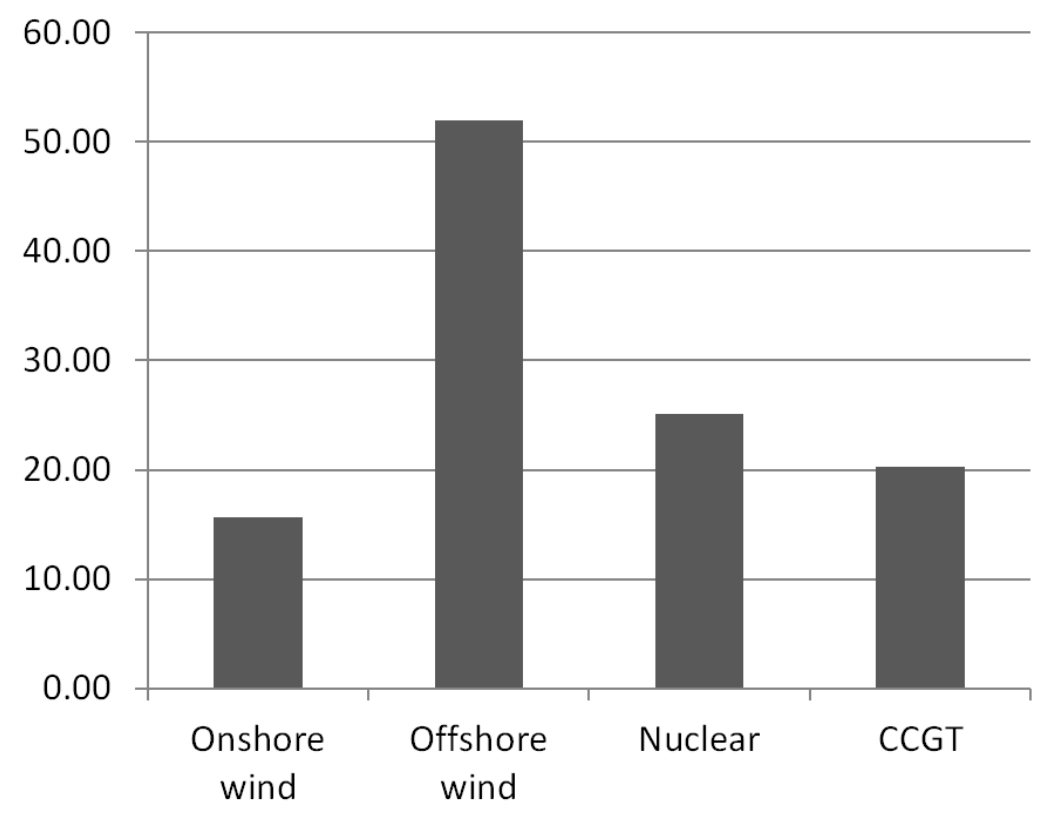

Figure 2: MAE by generation technology.

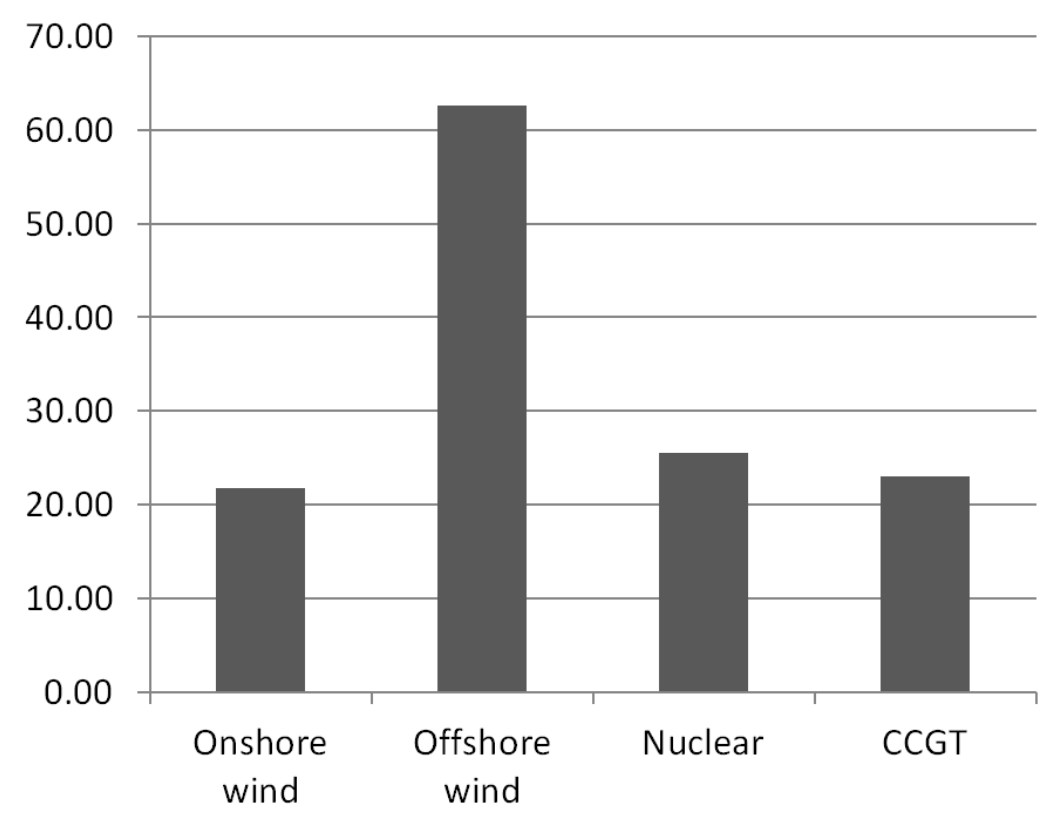

Figure 3: RMSE by generation technology. 


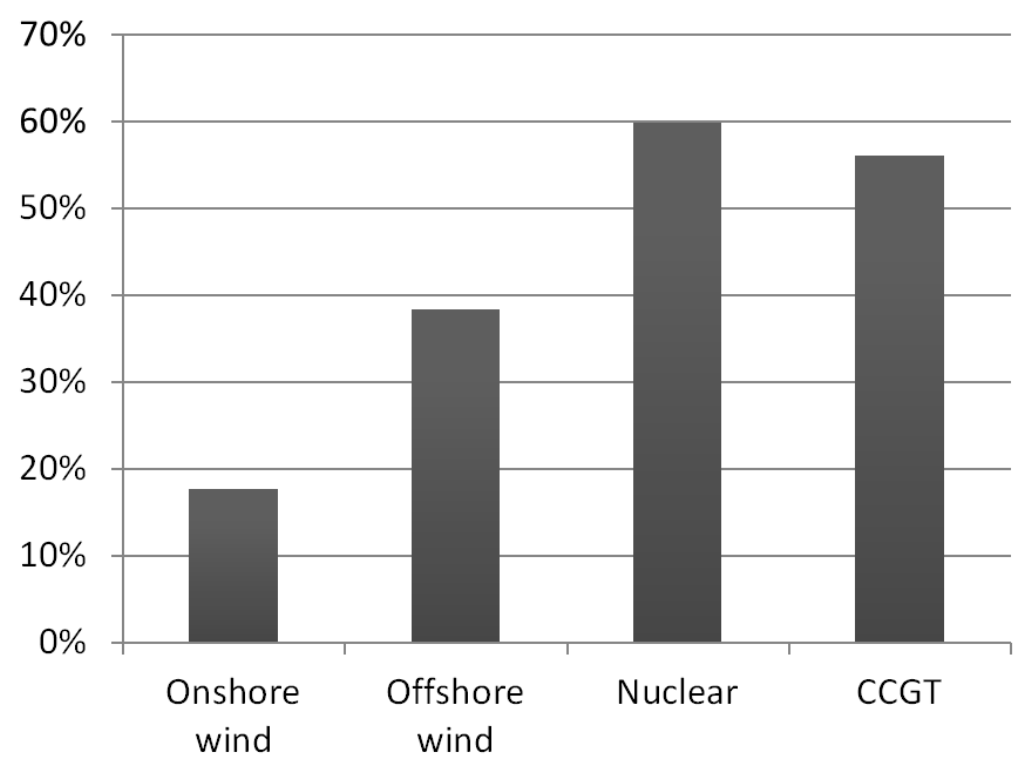

Figure 4: MAPE by generation technology.

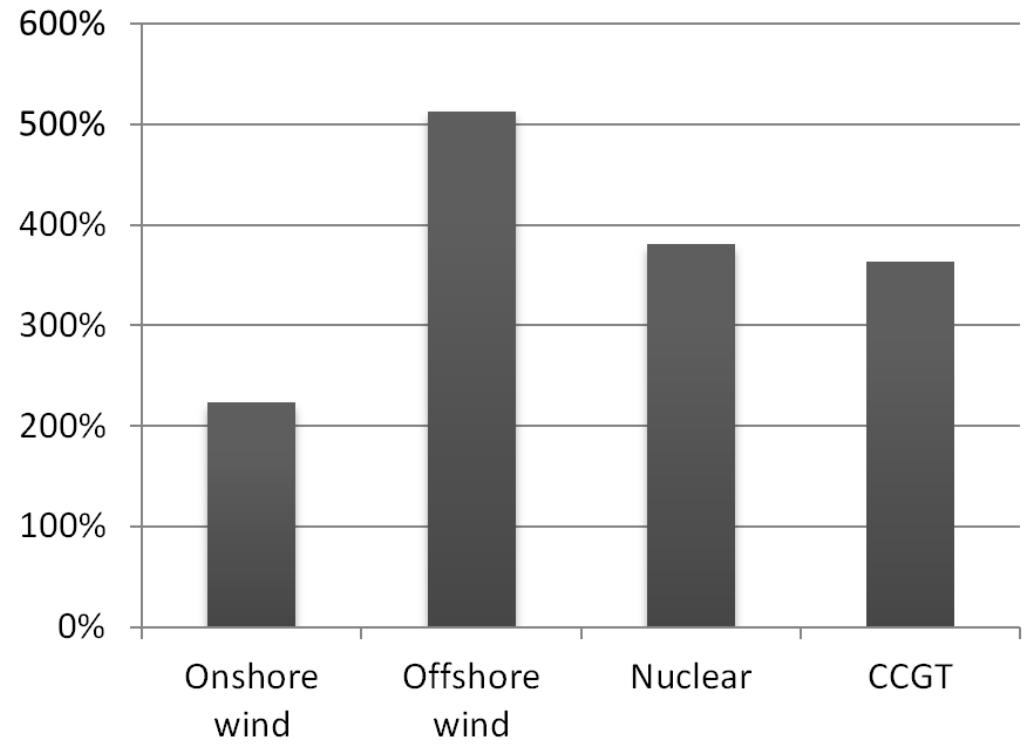

Figure 5: RMSPE by generation technology. 